\title{
EFEITOS DE UM PROGRAMA DE EXERCÍCIOS AQUÁTICOS EM CRIANÇAS COM DIABETES DO TIPO 1: RELATO DE CASO
}

Ana Elisa von Ah Morano ${ }^{1}$, Nayane Aparecida de Brito ${ }^{1}$, Daniela Bonfim Cortês ${ }^{2}$, Ana Paula Coelho Figueira Freire ${ }^{1}$, Francis Lopes Pacagnelli ${ }^{1}$, Deborah Cristina Gonçalves Luiz Fernani ${ }^{1}$, Maria Tereza Artero Prado ${ }^{1}$, Gabriela Andrade Piemonte ${ }^{1}$, Aline Duarte Ferreira ${ }_{1}$

${ }^{1}$ Universidade do Oeste Paulista - UNOESTE, Curso de Fisioterapia, Presidente Prudente, SP. ${ }^{2}$ Universidade Estadual Paulista - UNESP, Curso de Pós-graduação, Presidente Prudente, SP. Email: aline@unoeste.br

\section{RESUMO}

O objetivo foi relacionar o comportamento do nível glicêmico de crianças com diabetes mellitus tipo 1 com um programa de exercícios aquáticos. Duas crianças com idade cronológica de 8 e 12 anos e diagnóstico clínico de diabetes mellitus tipo 1 foram submetidas a um protocolo de exercícios aquáticos. 0 protocolo foi executado duas vezes por semana. As crianças foram avaliadas inicialmente e reavaliadas após as 18 sessões de treinamento quanto aos dados antropométricos e sinais vitais. A glicemia periférica foi verificada no início e fim de cada sessão. Após a execução do protocolo, observou-se redução dos níveis diários de glicemia das crianças, bem como a diminuição da necessidade de correção glicêmica por meio da insulina de ação rápida. As evidências apresentadas apoiam o uso de exercícios aquáticos como uma modalidade terapêutica a ser incorporada no processo de tratamento de crianças DM1, como uma prática não medicamentosa para o controle glicêmico.

Palavras-chave: Hidroterapia, atividade física, infância, Índice glicêmico, diabetes

\section{THE EFFECTS OF AN AQUATIC TRAINING PROGRAM IN CHILDREN WITH TYPE 1 DIABETES: CASE REPORT}

\begin{abstract}
The aim was to do a relation between the glucose level of children with type 1 diabetes and an aquatic training exercise program. Two children aged 8 and 12 years, with clinical diagnosis of type 1 diabetes mellitus (DM1), were submitted to an aquatic physical therapy exercises. The aquatic training was performed twice a week. The children were assessed and reassessed after the eighteen training sessions as the anthropometric data and vital signs. The peripheral glucose was checked before and at the end of each training session. After the protocol, was possible to notice a reduction on the glycemic daily results for both cases and a reduction on the use of rapid acting insulin. Supported by the evidence in this case report the practice of an aquatic training program as a therapeutic modality must be elected for the DM1 children treatment, as a non-medicinal practice for glycemic control.
\end{abstract}

Keywords: Hydrotherapy, physical activity, childhood, glycemic index, diabetes.

\section{INTRODUÇÃO}

O diabetes mellitus do tipo 1 (DM1) é conhecido como a doença da infância e da adolescência, autoimune, causada pela destruição de linfócitos $T$ mediadas por células pancreáticas $\beta$ e da deficiência de insulina, o que acarreta aumento da glicemia circulante ${ }^{1}$. O diagnóstico é geralmente feito na primeira década de vida e a criança passa a fazer uso da insulina para sua sobrevivência. Os sintomas mais frequentes que auxiliam a concluir o diagnóstico da doença são a sensação de sede constante, vontade de urinar diversas vezes ao dia, fome frequente e perda de peso ${ }^{2}$.

A prevalência de crianças com DM1 no Brasil com idades menores de 14 anos são de $4 / 10$ mil e $8 / 100$ mil habitantes $^{3}$. Considerada uma doença crônica de alta prevalência, o diabetes mellitus tornou-se um problema de saúde pública no Brasil, com incidência de DM1 aproximadamente $3 \%$ ao ano em crianças, o que totaliza mais de 200 casos por dia ${ }^{4}$. 
Há evidências científicas sobre os benefícios da regularidade na prática de exercícios físicos sobre o estado geral e metabolismo de crianças e adolescentes com DM1, cuja capacidade aeróbica melhorada também influencia no controle do índice glicêmico, reduzindo as doses diárias de insulina e riscos de complicações graves ${ }^{5}$. Em função do maior consumo de glicose pelos músculos estimulados pela prática de exercícios físicos, ocorre a redução da dose de insulina de ação rápida, em geral usada como fator de correção pré-refeição ${ }^{6}$.

Sabe-se que durante o tratamento de crianças com DM1, é crucial a prevenção de complicações crônicas como as doenças microvasculares, nefropatias, neuropatias diabéticas e retinopatias ${ }^{7}$. Com isso, a prática regular de exercícios físicos proporcionam melhores valores dos níveis de glicemia e pressão arterial, além dos efeitos benéficos no controle do peso, perfil das lipoproteínas e sensibilidade à insulina, o que auxilia a diminuição do risco de tais complicações ${ }^{8}$.

Dentre os tipos de exercícios físicos, os que são realizados em meio aquático estão sendo cada vez mais indicados, em razão dos seus efeitos benéficos nas diferentes variáveis da aptidão física, como a melhora da capacidade aeróbica, dos níveis de força e de flexibilidade, além da melhora na composição corporal ${ }^{9}$. Deste modo, como tratamento para o DM1, recomendam-se exercícios aeróbicos com duração de 30 a 90 minutos $^{10}$.

Sendo assim, o objetivo desse estudo foi relacionar o comportamento do nível glicêmico de crianças com DM1 com um programa de exercícios aquáticos de baixa intensidade.

\section{MÉTODOS}

Estudo de delineamento descritivo, do tipo longitudinal, realizado em uma piscina terapêutica de uma clínica escola de Fisioterapia de uma Universidade privada na cidade de Presidente Prudente, SP. A pesquisa foi aprovada pelo Comitê de Ética e Pesquisa envolvendo seres humanos da Universidade do Oeste Paulista e está de acordo com a resolução 466/2012 do CONEP (CAAE 40073514.7.0000.5515/2370). Os pais assinaram o termo de consentimento livre e esclarecido do representante legal e as crianças termo de assentimento. A execução do protocolo de exercícios aquáticos ocorreu entre os meses de maio e julho de 2015. Os exercícios foram realizados duas vezes por semana com duração de 45 minutos, totalizando 18 sessões.

Caso 1: A.B, 12 anos, sexo feminino, descobriu o DM1 há seis meses. A criança fazia uso de aplicações subcutânea de insulina/dia, de 4 unidades do tipo NPH (Neutral Protamine Hagedorn) e aplicação corrigida do tipo regular (acima de $150 \mathrm{mg} / \mathrm{dl}$ ) somente quando necessário.

Caso 2: R.M, oito anos, sexo masculino, descobriu o DM1 há um ano e dois meses. A criança fazia uso de aplicações subcutânea de insulina/dia do tipo NPH (Neutral Protamine Hagedorn), de 10 unidades e aplicação corrigida do tipo regular (acima de $150 \mathrm{mg} / \mathrm{dL}$ ) somente quando necessário.

A queixa principal das crianças foi relacionada ao descontrole do nível glicêmico e a quantidade de insulina/dia para correção diária. Ambos tiveram sintomas de poliúria e polidpsia antes do diagnóstico.

Antes do início da execução do protocolo de exercícios aquáticos (Pré-treino) e após as 18 sessões (Pós-treino), as crianças foram avaliadas quanto aos dados antropométricos como peso e altura; índice de massa corporal (IMC/idade) de acordo com Sisvan ${ }^{13}$ e avaliação do perímetro de cintura $(P C)^{14}$. Os sinais vitais como a frequência cardíaca e respiratória, e avaliação da glicemia periférica (aparelho OptiumXceed) foram verificados no início e término de cada sessão. Foram administrados rodízios de aplicação da insulina de ação rápida nas crianças antes do exercício quando a glicemia apresentou valores superiores ou inferiores para a realização do exercício ${ }^{12}$.

O protocolo de exercícios aquáticos sempre foi executado com a participação das duas crianças envolvidas no estudo. As sessões foram iniciadas com controle respiratório para adaptação ao meio aquático, permanecendo em posição semi-sentada com imersão até os ombros. Nessa posição, realizavam expirações lentas e longas repetidas vezes ao longo de 30 segundos e na sequência, duas voltas de caminhada dentro da piscina com braços abertos contra a resistência da água. Cada sessão contou com a execução de exercícios aeróbicos e anaeróbicos: duas voltas na piscina de marcha de lado com passos largos; em posição ortostática com água no nível do processo xifóide do osso esterno, realizaram 20 repetições de flexão e extensão de cotovelo; 20 repetições do 
movimento de flexão e extensão de ombros bilateralmente com os cotovelos estendidos, antebraços pronados e punho em posição neutra segurando um slade. Simultâneo aos movimentos dos membros superiores, realizaram 0 movimento de adução e abdução das pernas, para aumentar a intensidade dos exercícios e promover o caráter lúdico à essas crianças.

Na sequência, em posição semi-sentada com imersão no nível dos ombros, cotovelos estendidos, antebraços e punhos em posição neutra, executaram 20 repetições de abdução horizontal dos ombros sob superfície da água, com início em adução indo até $90^{\circ}$ de abdução horizontal segurando um slade. Após, as crianças em posição ventral com as mãos apoiadas na borda realizaram movimentos de batidas de perna, três séries de um minuto cada até atingir a sensação de esforço máximo e intervalo ativo de 30 segundos de caminhada lenta na posição horizontal da piscina.

Depois, foram orientadas a executar 20 repetições de marcha estática levando a mão ao joelho oposto fletido de forma alternada e na sequência, realizaram movimentos de bicicleta com auxílio de slades por dois minutos. Passaram para a posição ortostática e, segurando na borda da piscina, fizeram duas séries de 20 repetições de agachamentos. Por fim, de frente para a piscina, as crianças subiram os dois primeiros degraus e retornaram de costas, realizando movimentos rápidos por quatro minutos. 0 Protocolo de treinamento foi adaptado para crianças e adolescentes por meio de um estudo previamente publicado na literatura segundo Resende ${ }^{(11)}$.

\section{RESULTADOS}

O Caso 1 compareceu integralmente, sem ausências, em todos os dias da atividade. Já o Caso 2, por motivos justificados faltou em duas sessões, e em outra sessão foi dispensado por apresentar no dia uma glicemia pré-treino acima dos parâmetros requeridos pela literatura, que ressalta que pacientes não podem apresentar glicemia menor que $100 \mathrm{mg} / \mathrm{dL}$ ou maior que 250 $\mathrm{mg} / \mathrm{dL}^{12}$.

A Tabela 1 representa a caracterização dos participantes, com destaque para os valores da glicemia periférica na avaliação inicial no Caso 1 e Caso 2 de 163 mg/dL e 114 mg/dL, respectivamente.

Tabela 1. Caracterização dos participantes.

\begin{tabular}{lcc}
\hline Avaliação Inicial & Caso 1 & Caso 2 \\
\hline Peso (kg) & 37,8 & 31,8 \\
Altura (cm) & 1,50 & 1,32 \\
IMC (kg/m $\left.{ }^{2}\right)$ & 16,57 & 18,27 \\
PC (cm) & 64 & 67 \\
GL (mg/dL) & 163 & 114 \\
\hline
\end{tabular}

IMC: índice de massa corporal; PC: perímetro de cintura; GL: glicemia periférica.

Após 18 sessões de treinamento aquático, o Caso 2 apresentou uma diminuição de dois centímetros do perímetro de cintura quando comparado ao valor da avaliação inicial. Já o IMC de ambos os casos permaneceu inalterado após a finalização do treinamento aquático; o Caso 1 classificado como eutrófico e o Caso 2 classificado como sobrepeso ${ }^{13}$.

Sobre a glicemia periférica avaliada antes e após cada sessão de treinamento aquático, foi evidenciada diminuição nos valores no pós-treino em comparação ao pré-treino, tanto do Caso 1 (Figura 1) quanto do Caso 2 (Figura 2).

Também foi realizado o acompanhamento diário do comportamento do nível glicêmico ao longo do período de treinamento aquático do Caso 1. Observou-se assim diminuição do uso da insulina de correção por meio da curva diária de controle do índice glicêmico (Figura 3).

A curva diária de controle do índice glicêmico evidenciou um caráter descendente da glicemia após o início do treinamento aquático, ainda que superior aos valores do pós-treino, é evidentemente inferior após o início das sessões.

Como limitações do estudo, no Caso 2 não foi possível esboçar um gráfico de controle diário da glicemia por ausência de dados fornecidos pelo responsável da criança. 


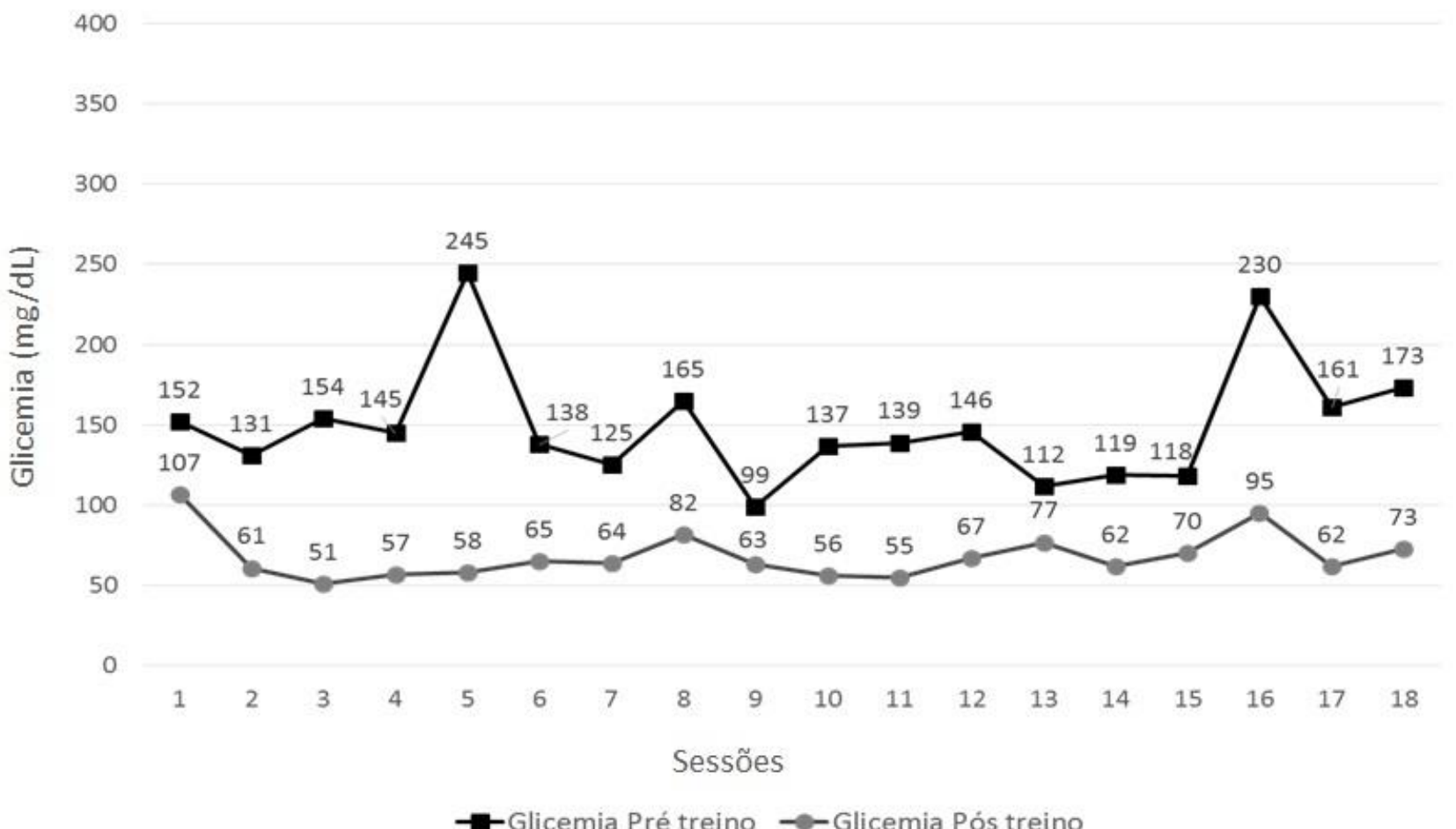

Figura 1. Comportamento da glicemia durante as 18 sessões de treinamento aquático, pré e pós-treino do Caso 1.

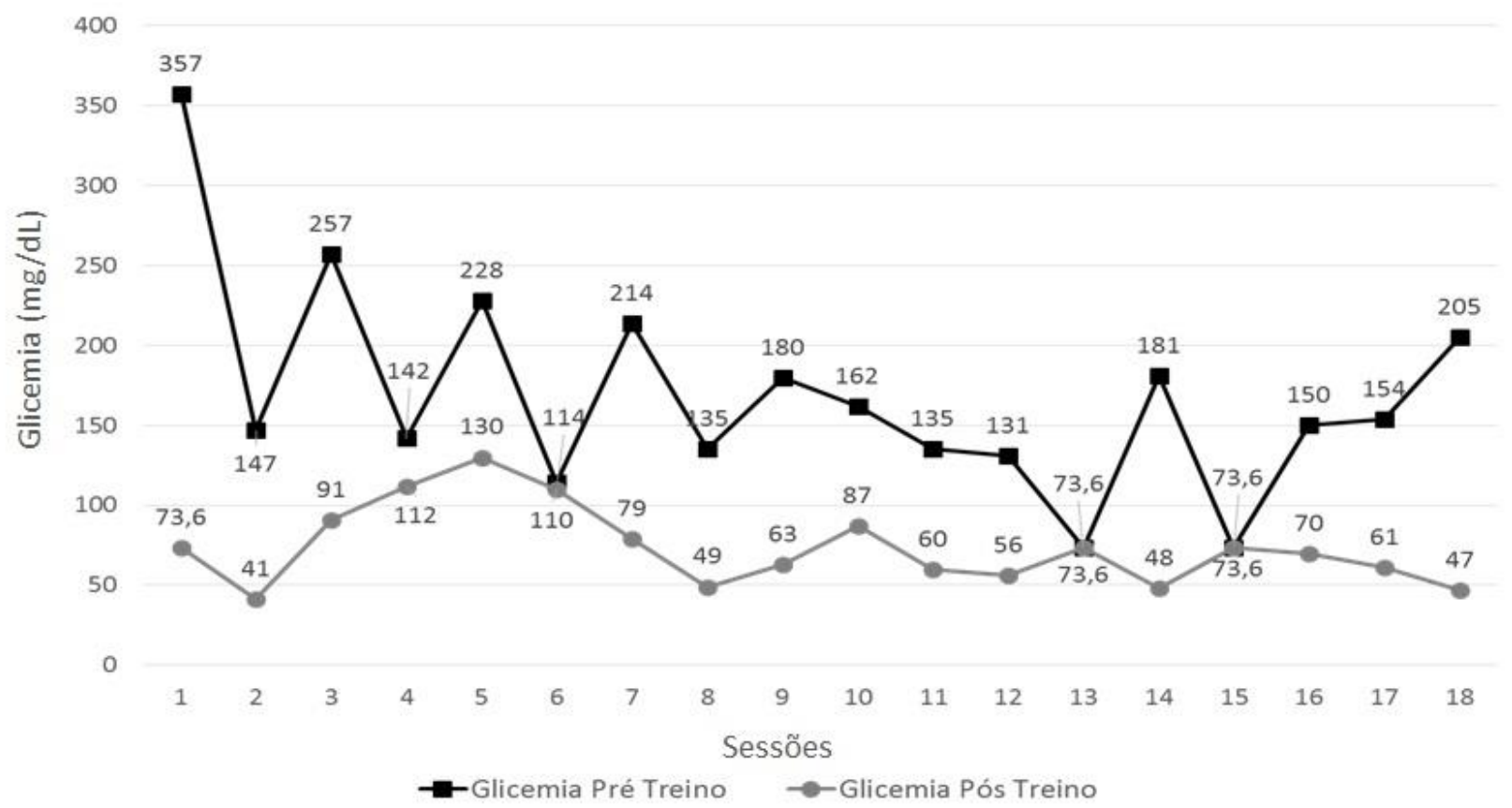

Figura 2. Comportamento da glicemia durante as 18 sessões de treinamento aquático, pré e pós-treino do Caso 2. 
250

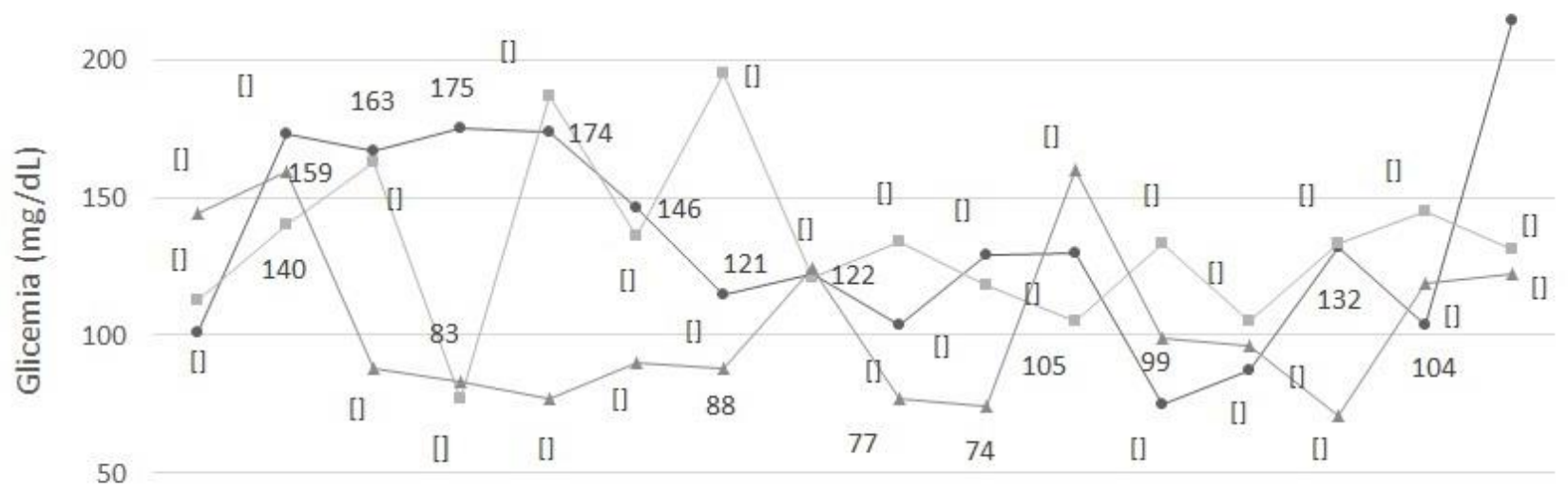

0

\begin{tabular}{|c|c|c|c|c|c|c|c|c|c|c|c|c|c|c|c|}
\hline 1 & 2 & 3 & 4 & 5 & 6 & 7 & 8 & 9 & 10 & 11 & 12 & 13 & 14 & 15 & 16 \\
\hline \multicolumn{16}{|c|}{ Sessões } \\
\hline & & & $\rightarrow-$ & jejum & & $\rightarrow-1$ & s prar & & 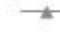 & Joite & & & & & \\
\hline
\end{tabular}

Figura 3. Curva diária de controle da glicemia pós sessão de exercício durante o período do treinamento aquático do Caso 1.

\section{DISCUSSÃO}

Após as 18 sessões do treinamento aquático foi observada redução dos níveis diários de glicemia em ambos os casos, bem como a diminuição da necessidade de correção glicemia por meio da insulina de ação rápida comprovada no Caso 1. Além do tratamento medicamentoso, a literatura traz que o exercício físico também apresenta indicação clínica no tratamento do diabetes mellitus por se tratar de um método eficaz na redução e manutenção dos níveis glicêmicos dentro da normalidade ${ }^{6}$. Entre as modalidades de exercícios físicos, a hidroterapia, além do ambiente descontraído e relaxante é considerado por muitos, em razão à sensação agradável em que o meio líquido proporciona durante a realização de exercícios ${ }^{9}$.

Nesse estudo notou-se que ao longo do treinamento aquático, o uso da insulina de correção pelos participantes em suas rotinas diárias foi diminuído. Segundo as recomendações médicas informadas pela família, a insulina de ação rápida deveria ser utilizada quando a glicemia estivesse maior que $150 \mathrm{mg} / \mathrm{dL}$ em um dos períodos de verificação de glicemia periférica (jejum, pós prandial ou noite). Durante o período do treinamento obteve-se vinte dias de anotações de controle diário do índice glicêmico realizado pelos familiares. Com isso, foi possível detectar um melhor controle glicêmico durante o período de treinamento aquático, e consequentemente menor frequência do uso da insulina de ação rápida. Os achados desse estudo corroboram com estudos previamente publicados na literatura a respeito do caráter depressor da glicemia provocado pela prática de exercícios físicos regulares ${ }^{15}$.

Outra limitação desse estudo refere-se ao número de participantes. Acredita-se que a baixa adesão dos pacientes muitas vezes ocorre por falta de incentivo dos programas de práticas esportivas e dificuldades de inclusão para populações específicas; além do fato da baixa divulgação ou não conhecimento dos benefícios que a prática regular de exercícios físicos traz para crianças e adolescentes com diabetes mellitus. Dessa forma sugere-se a realização de estudos com período de intervenção e amostra maiores.

Diante dos achados do presente estudo, foi possível notar que a prática regular, frequente e supervisionada de exercícios aquáticos influenciou no comportamento do nível glicêmico de crianças com DM1, e que esta modalidade de 
exercícios físicos pode ser recomendada para o tratamento da doença.

\section{AGRADECIMENTOS}

Ao curso de Fisioterapia e à Pró-Reitoria de Pesquisa e Pós-Graduação (PRPPG) da Unoeste pelo auxílio financeiro.

\section{CONFLITO DE INTERESSE}

Os autores declaram não haver qualquer potencial de conflito de interesse que possa interferir na imparcialidade deste trabalho científico.

\section{REFERÊNCIAS}

1. Ferrão ALM. Aspectos farmacológicos da insulinoterapia no diabetes Mellitus Tipo 1. Farmacologia da Universidade Federal de Lavras; 2013.

2. Heleno MGV, Vizzoto MM, Mazzotti T, Cressoni-Gomes R, Modesto SEF, Gouveia SRF. Acampamento de férias para jovens com Diabetes Mellitus Tipo I: Achados da abordagem psicológica. Bol Psicol. 2009;59(130):77-90.

3. Teles SAS, Schmid NF. Relação entre o perfil antropométrico e bioquímico em crianças e adolescentes com diabetes melito tipo 1 . Rev Paulista Pediatr. 2012;30(1):65-71. DOI: http://dx.doi.org/10.1590/S0103-

\section{0}

4. Oliveira ARS, Andrade CJN, Alves CAD, Mendes CNC. Prevalência e fatores de proteção para o controle glicêmico em crianças portadoras de diabetes melito tipo 1. Rev Ciên Méd Biol. 2015;15(3):317-22.

http://dx.doi.org/10.9771/cmbio.v15i3.18222

5. Robertson K, Riddell MC, Guinhouya BC, Adolfsson $P$, Hanas R. Exercise in children and adolescents with diabetes. Pediatr Diabetes. 2014;15(Suppl S20):203-23. DOI: http://dx.doi.org/10.1111/pedi.12176

6. Miculis CP, Mascarenhas LP, Boguszewski MC, Campos WD. Atividade física na criança com diabetes tipo. J Pediatr. 2010;86(4):271-8. DOI: http://dx.doi.org/10.1590/s0021-

\section{5}

7. Oliveira EV, Santos DF, Tetila AF, Silva DF, Gomes MJ, fell RF. Influência da pratica de atividades aquáticas na reposição de insulina e qualidade de vida no diabético tipo 1- Relato de Caso. Coll Vitae. 2012;4:176-83.

8. Shin KO, Moritani T, Woo J, Jang KS, Bae JY, Yoo J, Kang S. Exercise training improves cardiac autonomic nervous system activity in type 1 diabetic children. J Phys Ther Sci. 2014;26(1): 111-5. DOI: http://dx.doi.org/10.1589/ipts.26.111 9. Alberton LC, Kruel LFM. Influência da imersão nas respostas cardiorrespiratórias em repouso. Revista brasileira de medicina do esporte. 2009;15(3):228-32.

DOI:

http://dx.doi.org/10.1590/s151786922009000300013

10. Serrabulho L, Matos M, Nabais, Raposo J. A saúde e os estilos de vida com jovens adultos com diabetes tipo 1. Rev Port Diabetes. 2013;2:60-71.

11. Resende SM, Rassi CM, Viana FP. Efeitos da hidroterapia na recuperação do equilíbrio e prevenção de quedas em idosas. Rev Bras Fisioter. 2008;12(1):57-63. DOI: http://dx.doi.org/10.1590/s1413-

35552008000100011

12. Milech A, Angelucci AP, Golbert A, Carrilho AJF, Ramalho AC, Sachs A. Diretrizes da Sociedade Brasileira de Diabetes 2009. 3ed. Itapevi: Sociedade brasileira de diabetes; 2009.

13. Sisvan (Sistema de Vigilância Alimentar e Nutricional). Curvas de Crescimento da Organização Mundial da Saúde OMS. 2007 Disponível em: http://nutricao.saude.gov.br/sisvan.php?conteud o=curvas cresc oms

14. Reilly JJ, Dorosty, AR, Emmett PM. Identification of the obese child: adequacy of the body mass index for clinical practice and epidemiology. Int J Obesity, 2000;24(12):1623-7. DOI: http://dx.doi.org/10.1038/sj.ijo.0801436

15. Arrantes GN, Santos ACl, Navarro F. Influência do exercício físico combinado (aeróbio e de força) na necessidade de insulina exógena em indivíduos diabéticos tipo I. RBPFEX. 2011;3(15):284-294.

Recebido para publicação em 19/08/2016

Revisado em 22/08/2016

Aceito em 05/09/2016 